Pacific

Journal of

Mathematics

SUPERCONNECTIONS AND PARALLEL TRANSPORT

FLORIN DUMITRESCU 


\title{
SUPERCONNECTIONS AND PARALLEL TRANSPORT
}

\author{
FLORIN DUMITRESCU
}

\begin{abstract}
This note addresses the construction of a notion of parallel transport along superpaths arising from the concept of a superconnection on a vector bundle over a manifold $M$. A superpath in $M$ is, loosely speaking, a path in $M$ together with an odd vector field in $M$ along the path. We also develop a notion of parallel transport associated with a connection (also know as covariant derivative) on a vector bundle over a supermanifold, which is a direct generalization of the classical notion of parallel transport for connections over manifolds.
\end{abstract}

\section{Introduction}

The problem of understanding geometrically superconnections arose in an attempt to give examples of supersymmetric 1|1-field theories à la Stolz-Teichner [2004] over a manifold. Such field theories are expected to constitute cocycles for a differential version of topological K-theory [Hopkins and Singer 2005; Bunke and Schick 2007], providing the appropriate frame to formulate, for example, local family versions of the celebrated index theorems. Though these problems are related, it is not clear at this point how to formulate this connection.

Recall the classical parallel transport of a connection on a vector bundle over a manifold. Let $E$ be a vector bundle over a manifold $M$, and $\nabla$ a connection on $E$. Given a path $\gamma:[0,1] \rightarrow M$ joining two points $x$ and $y$ in $M$, the connection allows to identify linearly the fiber $E_{x}$ over $x$ with the fiber $E_{y}$ over $y$, via parallel sections along the path, that is, sections that are constant along the path with respect to the pullback connection. These identifications are compatible with gluing paths and invariant under reparametrization. This independence of metric allows us to refer to connections as examples of topological 1-dimensional field theories over a manifold.

An analogous construction carries over to the category of vector bundles over supermanifolds. The peculiar feature of a supermanifold is that its functions can also anticommute. Locally, a supermanifold of dimension $(p, q)$ looks like $\mathbb{R}^{p \mid q}$, the space whose functions are smooth functions on $\mathbb{R}^{p}$ tensored with an exterior

MSC2000: primary 55N15, 53C05; secondary $81 \mathrm{~T} 60$.

Keywords: superconnections, parallel transport, supermanifolds, supersymmetric field theories. 
algebra in $q$ odd generators. A supermanifold is represented by its ( $\mathbb{Z} / 2$-graded) algebra of functions. For example, given a vector bundle $E$ over a manifold, the supermanifold $\Pi E$ has as functions the sections of the exterior bundle $\Lambda E^{*}$. In particular, the "odd tangent bundle" $\Pi T M$ of a manifold $M$ has as functions the sections of the bundle $\Lambda T^{*} M$, that is, differential forms on $M$.

A connection $\nabla$ on a vector bundle $E$ over a supermanifold $M$ is defined by the usual Leibniz property: $\nabla(f s)=d f \otimes s \pm f \nabla s$ for functions $f$ on $M$ and sections $s$ of $E$. Geometrically, such a connection gives rise to parallel transport along superpaths $\alpha: \mathbb{R}^{1 \mid 1} \rightarrow M$. Namely, a section along $\alpha$ is parallel if it is constant in the direction of the $o d d$ vector field $D=\partial_{\theta}+\theta \partial_{t}$ on $\mathbb{R}^{1 \mid 1}$. The resulting parallel transport by parallel sections along superpaths is compatible under gluing of superpaths and (conformal - see Section 3.2) reparametrizations of superpaths.

Next, we want to interpret superconnections geometrically (as parallel transport). Recall [Quillen 1985; Berline et al. 1992] that a superconnection on a $\mathbb{Z} / 2$ graded vector bundle $E$ over a manifold $M$ is an odd degree first order differential operator defined on the space $\Omega^{*}(M, E)$ of sections of the bundle $\Lambda T^{*} M \otimes E$ over $M$, with $\mathbb{A}: \Omega^{*}(M, E) \rightarrow \Omega^{*}(M, E)$ satisfying the (graded) Leibniz rule. A can be written $\mathbb{A}=\mathbb{A}_{0}+\mathbb{A}_{1}+\mathbb{A}_{2}+\cdots$, with $\mathbb{A}_{1}$ a grading-preserving connection on $E$, and $\mathbb{A}_{i}$ for $i \neq 1$ is given by multiplication by some form $\omega_{i} \in \Omega^{i}(M$, End $E)$.

We begin with a notion of parallel transport associated to a grading preserving connection $\nabla$ on a $\mathbb{Z} / 2$-graded vector bundle $E$ over a manifold $M$ and an End $E$ valued form $A \in \Omega^{*}(M \text {, End } E)^{\text {odd }}$ on $M$. Then $A$ can be viewed as a section of the endomorphism bundle End $\pi^{*} E$, where $\pi: \Pi T M \rightarrow M$ is the map that on functions is the inclusion of 0 -forms into the space of differential forms. Let $c: \mathbb{R}^{1 \mid 1} \rightarrow M$ be a superpath in $M$. (We think, as is customary, in families of superpaths — see [Witten 1999; Freed 1999].) Then $c$ lifts naturally (see Section 4.1) to a superpath $\tilde{c}: \mathbb{R}^{1 \mid 1} \rightarrow \Pi T M$. A section $\psi$ along $c$ is parallel if

$$
\left(c^{*} \nabla\right)_{D} \psi-\left(\tilde{c}^{*} A\right) \psi=0 .
$$

The resulting parallel transport is compatible under gluing of superpaths and converges (by an inverse adiabatic limit process) to the parallel transport associated to the connection $\nabla$. As a corollary to this construction we obtain a parallel transport corresponding to a superconnection $\mathbb{A}=\mathbb{A}_{1}+A$, by viewing $\mathbb{A}_{1}$ as a graded connection and $A \in \Omega^{*}(M \text {, End } E)^{\text {odd }}$ as above.

\section{A short introduction to supermanifolds}

We give in this section a brief introduction to the theory of supermanifolds. The subject was introduced and developed by Leĭtes [1980], Bernstein, and Manin [1988]. A good expository reference is Deligne and Morgan [1999]. The reader 
can also consult Varadarajan [2004]. We spend here some time talking about integration of vector fields on supermanifolds, since we could not find a detailed account on the topic in the literature.

Start with the ringed space $\mathbb{R}^{p \mid q}=\left(\mathbb{R}^{p}, \mathscr{C}_{\mathbb{R}^{p}}^{\infty} \otimes \Lambda\left[\theta_{1}, \ldots, \theta_{q}\right]\right)$. A supermanifold $M$ of dimension $p \mid q$ is a pair $\left(|M|, \mathcal{O}_{M}\right)$ with $|M|$ a topological space and $\mathrm{O}_{M}$ a sheaf on $|M|$ of $\mathbb{Z} / 2$-graded algebras that locally is isomorphic to $\mathbb{R}^{p \mid q} .|M|$ is called the underlying space of $M$ and $O_{M}$ is the structure sheaf of $M$. The odd functions generate a nilpotent ideal $\mathscr{F}$ of $\mathcal{O}_{M}$ and $\left(|M|, \mathrm{O}_{M} / \mathscr{f}\right)$ is a smooth manifold of dimension $p$, called the reduced manifold $M_{\text {red }}$ of $M$.

A morphism of supermanifolds $f: M \rightarrow N$ is a pair $f=\left(|f|, f^{\sharp}\right)$ consisting of a continuous map $|f|:|M| \rightarrow|N|$ and a map $f^{\sharp}: \mathcal{O}_{N} \rightarrow|f|_{*} \mathcal{O}_{M}$ of sheaves of $\mathbb{Z} / 2$-graded algebras. For example, there is a canonical morphism $i: M_{\text {red }} \hookrightarrow M$, which on the underlying spaces is the identity; the map on sheaves is the projection $i^{\sharp}: \mathrm{O}_{M} \rightarrow \mathrm{O}_{M} / \mathscr{F}$. A morphism $f: M \rightarrow N$ induces a morphism between the corresponding reduced manifolds since it preserves the nilpotent ideal sheaves. A morphism of supermanifolds is uniquely determined by the map induced on global sections [Kostant 1977, p. 208]. So, instead of a map of sheaves, we will consider just the map induced on their global sections. Supermanifolds are examples of ringed spaces and the category $\mathbf{S M}$ of supermanifolds embeds fully faithfully in the category of ringed spaces.

An important source of examples of supermanifolds comes from vector bundles. To any vector bundle $E$ over a manifold $M_{0}$ one can associate a supermanifold $\Pi E=\left(M_{0}, \mathcal{O}_{\Pi E}\right)$, where $\mathcal{O}_{\Pi E}$ is the sheaf of sections of $\Lambda E^{*}$. This defines a functor

\section{$S: \mathbf{V B} \longrightarrow \mathbf{S M}: E \longmapsto \Pi E$}

from the category of vector bundles to the category of supermanifolds. There is also a functor $V$ going the other direction. Namely, let $M=\left(M_{0}, \mathcal{O}_{M}\right)$ be a supermanifold. Then $\left(\mathscr{F} / \mathscr{F}^{2}\right)^{*}$ is a locally free sheaf on $M_{0}$, where $\mathscr{F}$ is the nilpotent ideal of $O_{M}$, so it determines a vector bundle on $M_{0}$. We have that $V \circ S=\mathrm{id}$ and $S \circ V=$ id on isomorphism classes of objects. This doesn't assure an equivalence of categories though, since $S V$ fails to be the identity on morphisms (for instance, it maps the automorphism $\left(x, \theta_{1}, \theta_{2}\right) \mapsto\left(x+\theta_{1} \theta_{2}, \theta_{1}, \theta_{2}\right)$ of $\mathbb{R}^{1 \mid 2}$ to id). The category of supermanifolds is richer in morphisms. This relation between the categories is analogous to the one between graded rings (vector bundles) and filtered rings (supermanifolds).

2.1. The "functor of points" viewpoint. In the superworld one cannot talk properly about points on a supermanifold unless one refers to points on the reduced manifold. A more suitable approach is the lingo of $S$-points. Consider a supermanifold $M$. An $S$-point of $M$ for an arbitrary supermanifold $S$ is a map $S \rightarrow M$, 
and the $S$-points of $M$ is the set $M(S)=\mathbf{S M}(S, M)$. This is the approach physicists adopt in computations, which also resonates with our geometric intuition. One can think of an $S$-point as a family of points of $M$ parametrized by $S$. For example, as sets,

$$
M\left(\mathbb{R}^{0 \mid 0}\right)=\mathbf{S M}\left(\mathbb{R}^{0 \mid 0}, M\right)=|M| .
$$

If $T \stackrel{\alpha}{\longrightarrow} S$ is a map in $\mathbf{S M}$, there is a natural map $M(S) \rightarrow M(T): m \mapsto m \circ \alpha$. So $M$ determines a contravariant functor

$$
\mathbf{S M}^{\text {op }} \rightarrow \text { Sets }: S \mapsto M(S)
$$

called the functor of points of $M$. A map $f: M \rightarrow N$ of supermanifolds determines a natural transformation $\mathbf{S M}(\cdot, M) \rightarrow \mathbf{S M}(\cdot, N)$. The converse of this is also true, and forms the content of Yoneda's lemma. This means that to give a map $M \rightarrow N$ amounts to giving maps of sets $M(S) \rightarrow N(S)$, natural in $S$.

We can therefore think of a supermanifold $M$ as a representable functor $\mathbf{S M}^{\mathrm{op}} \rightarrow$ Sets, such a functor determining $M$ uniquely up to isomorphism. For example, if $M, N$ are two supermanifolds, their product $M \times N$ can be interpreted as the supermanifold representing the functor

$$
S \mapsto \mathbf{S M}(S, M) \times \mathbf{S M}(S, N) .
$$

An arbitrary contravariant functor $\mathbf{S M} \rightarrow$ Sets will be called a generalized supermanifold. The category $\mathbf{S M}$ of supermanifolds embeds fully faithful into the category GSM of generalized supermanifolds. Consider, for example, two supermanifolds $M, N$, and define the generalized supermanifold

$$
\underline{\mathbf{S M}}(M, N): \mathbf{S M} \rightarrow \text { Sets }: S \longmapsto \mathbf{S M}(S \times M, N) .
$$

If $\underline{\mathbf{S M}}(M, N)$ is an ordinary supermanifold, then we have the adjunction formula

$$
\mathbf{S M}(S, \underline{\mathbf{S M}}(M, N)) \cong \mathbf{S M}(S \times M, N) .
$$

2.2. The tangent sheaf and tangent vectors. The analogue of the tangent bundle in classical differential geometry is the tangent sheaf $\mathscr{T} M$ defined as the sheaf of graded derivations of $\mathrm{O}_{M}$, that is, for $U \subseteq|M|$,

$\mathscr{T} M(U)=\left\{X: \mathscr{O}_{M}(U) \rightarrow \mathscr{O}_{M}(U)\right.$ linear $\left.\mid X(f g)=X(f) g+(-1)^{p(X) p(f)} f X(g)\right\}$.

Here $p(X)=0$ or 1 according to whether $X$ is an even or, respectively, odd vector field on $U$, and similarly $p(f)=0$ or 1 , for $f$ an even or, respectively, odd function on $M . \mathscr{T} M$ is then a locally free $\mathcal{O}_{M}$-module of rank $(p, q)$, the dimension of the supermanifold $M$. Sections of $\mathscr{T} M$ are the vector fields on $M$. For $X$ and $Y$ vector fields on $M$, define as usual their Lie bracket $[X, Y]$ by

$$
[X, Y](f)=X(Y(f))-(-1)^{p(X) p(Y)} Y(X(f)) \quad \text { for } f \in \mathscr{C}^{\infty}(M)=\mathcal{O}_{M}(|M|) .
$$


For example, consider on $\mathbb{R}^{1 \mid 1}$ the vector field $D=\partial_{\theta}+\theta \partial_{t}$. Then, one can check that

$$
D^{2}=\frac{1}{2}[D, D]=\partial_{t} .
$$

Similarly, if $Q=\partial_{\theta}-\theta \partial_{t}$, then

$$
Q^{2}=\frac{1}{2}[Q, Q]=-\partial_{t} .
$$

For $m \in M(S)$ an $S$-point of $M$, define the tangent space at $m$ to $M$ by

$$
T M_{m}=\left\{v: \mathscr{C}^{\infty}(M) \rightarrow \mathscr{C}^{\infty}(S) \mid v(f g)=v(f) m^{\sharp}(g)+(-1)^{p(v) p(f)} m^{\sharp}(f) v(g)\right\} .
$$

For $m \in M$ an ordinary point, we get the usual definition of the tangent space at $m$.

2.3. Geometric structures on $(1,1)$-supermanifolds. Suppose $Y$ is a $(1,1)$-supermanifold. Then the tangent sheaf $\mathscr{T} Y$ is a locally free $\mathcal{O}_{Y}$-module of rank $(1,1)$ : if $(t, \theta)$ are local coordinates on $Y$ then $\left\{\partial_{t}, \partial_{\theta}\right\}$ forms a local basis for $\mathscr{T} Y$. A conformal structure on $Y$ is a rank $(0,1)$ distribution $\mathscr{D}$, that is, a rank $(0,1)$ subsheaf of the tangent sheaf $\mathscr{T} Y$, that fits into the following short exact sequence of sheaves:

$$
0 \rightarrow \mathscr{D} \longrightarrow \mathscr{T} Y \longrightarrow \mathscr{D}^{\otimes 2} \rightarrow 0 \text {. }
$$

A euclidean (metric) structure on $Y$ is given by the choice of an odd vector field $D$ generating an odd distribution $\mathscr{D}$ as above. For example, on $\mathbb{R}^{1 / 1}$ consider the vector field $D=\partial_{\theta}+\theta \partial_{t}$. Then $D$ defines a metric structure on $\mathbb{R}^{1 \mid 1}$, called the standard metric structure on $\mathbb{R}^{1 \mid 1}$. Also $\mathscr{D}=\langle D\rangle$, the distribution generated by $D$, defines a conformal structure on $\mathbb{R}^{1 \mid 1}$ : indeed, the square of $D$ is $D^{2}=\partial_{t}$, and the

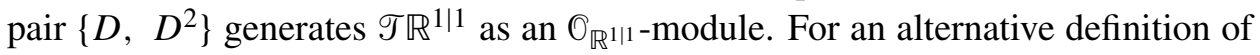
metric structures see [Stolz and Teichner 2004, Section 3.2].

2.4. The super Lie group $\mathbb{R}^{\mathbf{1} \mid \mathbf{1}}$. Super Lie groups are the super analogue of Lie groups in differential geometry. Let for example $\mathbb{R}^{1 \mid 1}$ be the super Lie group with the multiplication map $m: \mathbb{R}^{1 \mid 1} \times \mathbb{R}^{1 \mid 1} \rightarrow \mathbb{R}^{1 \mid 1}$, which defined on $S$-points by

$$
(t, \theta),\left(t^{\prime}, \theta^{\prime}\right) \stackrel{m_{S}}{\longmapsto}\left(t+t^{\prime}+\theta \theta^{\prime}, \theta+\theta^{\prime}\right) .
$$

Here $t$ and $t^{\prime}$ are even functions on $S, \theta$ and $\theta^{\prime}$ are odd functions on $S$, and so on. Observe that $\theta \theta^{\prime}$ is an even function on $S$. The map $m$ defines a group multiplication on $\mathbb{R}^{1 \mid 1}$, with identity given by $(0,0) \in \mathbb{R}^{1 \mid 1}$ and the inverse map given by $(t, \theta) \mapsto(-t,-\theta)$. This is the group structure on $\mathbb{R}^{1 \mid 1}$ that we will mostly use in this paper; therefore we are going to call it the standard group structure on $\mathbb{R}^{1 \mid 1}$.

As in the classical theory of Lie groups, we can consider left (right) invariant vector fields and identify them with the tangent space at the identity $e \in G$. Let $X$ be a vector field on a super Lie group $G$, that is, a graded derivation 
$X: \mathscr{C}^{\infty}(G) \rightarrow \mathscr{C}^{\infty}(G) . X$ is left-invariant if the diagram

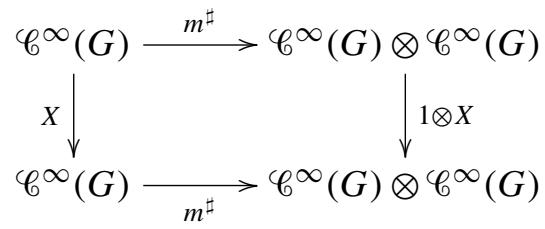

commutes, that is, $m^{\sharp} \circ X=(1 \otimes X) \circ m^{\sharp}$. The diagram expresses the fact that $X$ is an infinitesimal right translation.

Consider, for example, $\mathbb{R}^{1 \mid 1}$ with the standard group structure defined above. Let $Q$ be the vector field on $\mathbb{R}^{1 \mid 1}$ given by $Q=\partial_{\theta}-\theta \partial_{t}$, in coordinates $(t, \theta)$ on $\mathbb{R}^{1 \mid 1}$. Let us show that $Q$ is left-invariant. We need to check the commutativity of the diagram

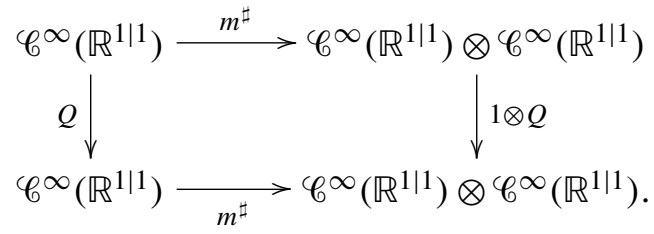

This is verified by looking at the two commutative diagrams
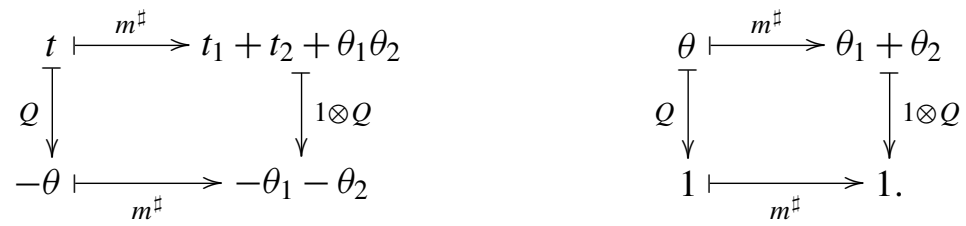

Analogously, a vector field $X$ on a supermanifold $M$ is right-invariant if $m^{\sharp} \circ X=$ $(X \otimes 1) \circ m^{\sharp}$. One can check for example that the vector field $D=\partial_{\theta}+\theta \partial_{t}$ is a right-invariant vector field on $\mathbb{R}^{1 \mid 1}$.

\subsection{Some identifications.}

Lemma 2.1. Let $M$ be an ordinary manifold. Then, we can identify

$$
\underline{\mathbf{S M}}\left(\mathbb{R}^{0 \mid 1}, M\right) \cong \Pi T M,
$$

where $\Pi$ TM is the odd tangent bundle of $M$.

Proof. We want to show that we have isomorphisms

$$
\Psi_{S}: \mathbf{S M}\left(S \times \mathbb{R}^{0 \mid 1}, M\right) \rightarrow \mathbf{S M}(S, \Pi T M),
$$

natural in $S$, where $S$ is an arbitrary supermanifold. The left hand side is the set of grading-preserving maps of $\mathbb{Z} / 2$-algebras

$$
\varphi: \mathscr{C}^{\infty}(M) \rightarrow \mathscr{C}^{\infty}\left(S \times \mathbb{R}^{0 \mid 1}\right)=\mathscr{C}^{\infty}(S)[\theta] .
$$


If we write $\varphi(f)=\varphi_{1}(f)+\theta \varphi_{1}(f)$, for $f \in \mathscr{C}^{\infty}(M)$, then the fact that $\varphi(f g)=$ $\varphi(f) \varphi(g)$ is equivalent to the conditions

$$
\varphi_{1}(f g)=\varphi_{1}(f) \varphi_{1}(g) \quad \text { and } \quad \varphi_{2}(f g)=\varphi_{2}(f) \varphi_{1}(g)+(-1)^{p(f)} \varphi_{1}(f) \varphi_{2}(g) .
$$

The first condition is equivalent to $\varphi_{1}=a^{\sharp}$ for some $a: S \rightarrow M$. The second tells us that $\varphi_{2}$ is an odd tangent vector at $a \in M(S)$, that is, $\varphi_{2}=X_{a} \in T M_{a}$. Therefore the left hand side is

$\mathbf{S M}\left(S \times \mathbb{R}^{0 \mid 1}, M\right)=\left\{\right.$ pairs $\left(a, X_{a}\right) \mid a \in M(S), X_{a} \in T M_{a}, X_{a}$ odd $\}$.

$\mathbf{S M}(S, \Pi T M)$, at right, is the set of $\mathbb{Z} / 2$-graded algebra maps $\Omega^{*}(M) \rightarrow \mathscr{C}^{\infty}(S)$. Such maps are determined by their restriction to 0 -forms (functions) and 1-forms (more specifically, 1-forms of the type $d f$ for $f \in \mathscr{C}^{\infty}(M)$ ). Define then $\Psi_{S}\left(a, X_{a}\right)$ to be the map $S \rightarrow \Pi T M$ determined by defining it on functions $f \in \mathscr{C}^{\infty}(M)$ by $a^{\sharp}(f) \in \mathscr{C}^{\infty}(S)$ and on forms $d f$ by $X_{a}(f)$. One can easily check that $\Psi_{S}$ is welldefined, bijective, and natural in $S$.

Let $T: \Pi T M \times \mathbb{R}^{0 \mid 1} \rightarrow \Pi T M$ be the map which on functions is given by $\Omega^{*}(M) \ni \omega \mapsto \omega+\theta d \omega \in \Omega^{*}(M)[\theta]$. Consider also the map

$$
\mu: \underline{\mathbf{S M}}\left(\mathbb{R}^{0 \mid 1}, M\right) \times \mathbb{R}^{0 \mid 1} \rightarrow \underline{\mathbf{S M}}\left(\mathbb{R}^{0 \mid 1}, M\right)
$$

that is defined on $S$-points

$$
\mathbf{S M}\left(S \times \mathbb{R}^{0 \mid 1}, M\right) \times \mathbf{S M}\left(S, \mathbb{R}^{0 \mid 1}\right) \rightarrow \mathbf{S M}\left(S \times \mathbb{R}^{0 \mid 1}, M\right)
$$

by $(\varphi, \eta) \mapsto \varphi \circ(1 \times m) \circ(1 \times \eta \times 1) \circ(\Delta \times 1)$, where $m$ is the group composition map on $\mathbb{R}^{0 \mid 1}$. The maps $T$ and $\mu$ define an action of $\mathbb{R}^{0 \mid 1}$ on the corresponding spaces.

Lemma 2.2. The map defined in the previous lemma, $\Psi: \underline{\mathbf{S M}}\left(\mathbb{R}^{0 \mid 1}, M\right) \rightarrow \Pi T M$, is $\mathbb{R}^{0 \mid 1}$-equivariant.

Proof. We want to show the commutativity of

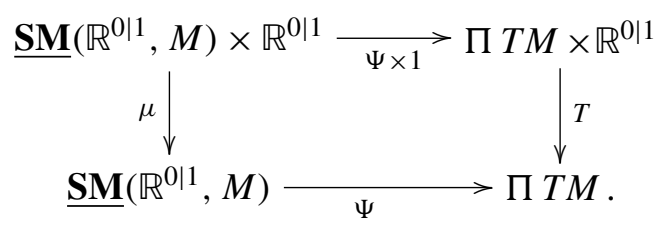


We need that, for each supermanifold $S$, natural in $S$, the commutativity of

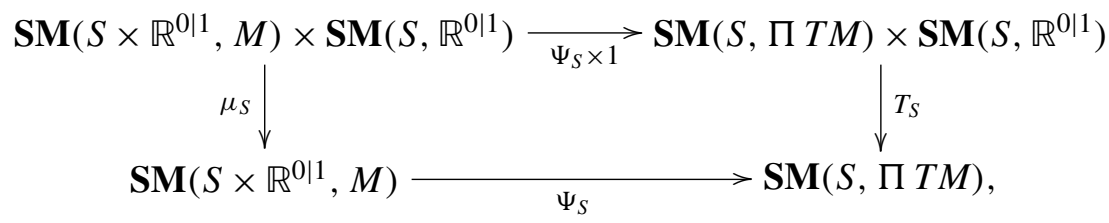

or, in terms of functions, we need to have

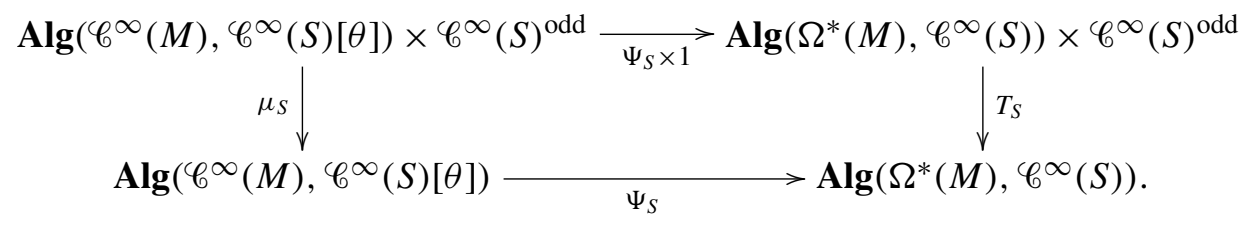

For $a \in M(S)$ and $X_{a} \in T M_{a}$, denote by $\left(a, X_{a}\right) \in \mathbf{A} \lg \left(\Omega^{*}(M), \mathscr{C}^{\infty}(S)\right)$ the map determined by $f \mapsto a^{\sharp}(f)$ and $d f \mapsto X_{a}(f)$. (Compare the proof of the previous lemma.) Via the identification

$$
\operatorname{Alg}\left(\Omega^{*}(M), \mathscr{C}^{\infty}(S)\right) \times \mathscr{C}^{\infty}(S)^{\text {odd }}=\mathbf{A} \mathbf{I g}\left(\Omega^{*}(M)[\theta], \mathscr{C}^{\infty}(S)\right),
$$

the map

$$
T_{S}: \operatorname{Alg}\left(\Omega^{*}(M), \mathscr{C}^{\infty}(S)\right) \times \mathscr{C}^{\infty}(S)^{\text {odd }} \rightarrow \operatorname{Alg}\left(\Omega^{*}(M), \mathscr{C}^{\infty}(S)\right)
$$

evaluated at $\varphi=\left(\left(a, X_{a}\right), \tilde{\theta}\right) \in \mathbf{A} \lg \left(\Omega^{*}(M), \mathscr{C}^{\infty}(S)\right) \times \mathscr{C}^{\infty}(S)^{\text {odd }}$ is determined by saying that

$$
\begin{gathered}
f \stackrel{T^{\sharp}}{\longmapsto} f+\theta d f \stackrel{\varphi^{\sharp}}{\longrightarrow} a^{\sharp}(f)+\tilde{\theta} X_{a}(f)=: b^{\sharp}(f), \\
d f \stackrel{T^{\sharp}}{\longmapsto} d f \stackrel{\varphi^{\sharp}}{\longmapsto} X_{a}(f)=: X_{b}(f),
\end{gathered}
$$

where $b \in M(S)$ is defined by $b^{\sharp}(f)=a^{\sharp}(f)+\tilde{\theta} X_{a}(f)$ for $f \in \mathscr{C}^{\infty}(M)$.

On the other hand,

$$
\mu_{S}: \mathbf{S M}\left(S \times \mathbb{R}^{0 \mid 1}, M\right) \times \mathbf{S M}\left(S, \mathbb{R}^{0 \mid 1}\right) \rightarrow \mathbf{S M}\left(S \times \mathbb{R}^{0 \mid 1}, M\right)
$$

is defined by $\left(\alpha=\left(a, X_{a}\right), \eta\right) \mapsto \alpha \circ(1 \times m) \circ(1 \times \eta \times 1) \circ(\Delta \times 1)$, or, on functions, $\mu_{S}\left(\left(a, X_{a}\right), \tilde{\theta}\right)$ is given by

$$
\begin{aligned}
f & \stackrel{\alpha^{\sharp}}{\longmapsto} a^{\sharp}(f)+\theta X_{a}(f) \\
& \stackrel{1 \otimes m^{\sharp}}{\longmapsto} a^{\sharp}(f)+\left(\theta_{1}+\theta_{2}\right) X_{a}(f) \\
& \stackrel{1 \otimes v^{\sharp} \otimes 1}{\longmapsto} a^{\sharp}(f)+\left(\theta_{1}+\tilde{\theta}\right) X_{a}(f) \\
& \stackrel{\Delta^{\sharp} \otimes 1}{\longmapsto} a^{\sharp}(f)+\tilde{\theta} X_{a}(f)+\theta X_{a}(f)=b^{\sharp}(f)+\theta X_{b}(f) .
\end{aligned}
$$


Therefore we have

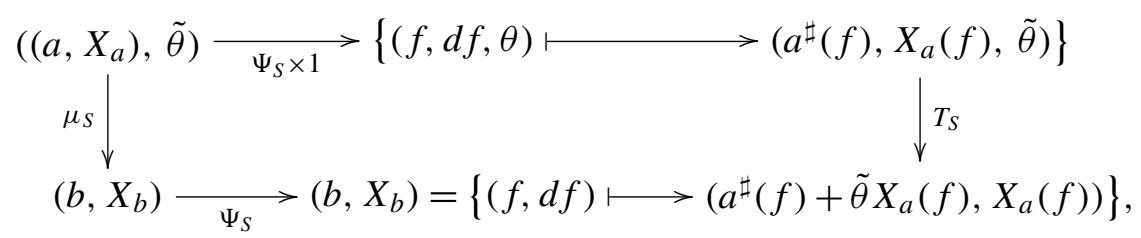

which verifies the commutativity of the above diagram. The lemma is proved.

2.6. Differential equations on supermanifolds. In what follows we will show that vector fields (even or odd) on supermanifolds can be integrated. We consider first the even case.

Lemma 2.3. Let $X$ be an even vector field on a compact supermanifold $M$ (that is, the underlying manifold is compact). Then there exists a unique map $c: \mathbb{R} \times M \rightarrow M$ satisfying the conditions

$$
\partial_{t} \circ c^{\sharp}=c^{\sharp} \circ X \text { and }\left.c\right|_{0 \times M}=\mathrm{id}_{M} .
$$

The map $c$ is called the flow of the vector field $X$.

Proof. The existence and uniqueness of a global solution follows from the existence and uniqueness of a local solution, since $M$ is compact. To solve the local problem, we can assume without loss of generality that $M=\mathbb{R}^{p \mid q}$. Let $x^{1}, \ldots, x^{p+q}$ be the coordinate functions on $\mathbb{R}^{p \mid q}$, with the first $p$ coordinates even and the last $q$ odd. We also write $\theta_{1}, \ldots, \theta_{q}$ for the last $q$ odd coordinates. Let $c^{i}$ be the image of $x^{i}$ under the map $c^{\sharp}$. Let us write

$$
c^{i}=\sum c_{J}^{i} \theta^{J} \quad \text { with } c_{J}^{i} \in \mathscr{C}^{\infty}\left(\mathbb{R} \times \mathbb{R}^{p}\right) .
$$

The vector field $X$ can be written $X=\sum_{1}^{p+q} a_{i} \partial_{x^{i}}$, where $a_{i}$ are even functions on $\mathbb{R}^{p \mid q}$ for $i=1, \ldots, p$ and odd functions on $\mathbb{R}^{p \mid q}$ for $i=p+1, \ldots, p+q$. We further write

$$
a_{i}=\sum a_{J}^{i} \theta^{J} \quad \text { with } a_{J}^{i} \in \mathscr{C}^{\infty}\left(\mathbb{R}^{p}\right)
$$

and with some of the $a_{J}^{i}$ possibly zero. The first condition above holds for a map $c: I \times M \rightarrow M$, with $I$ a small neighborhood of 0 , if and only if it holds when evaluated on the coordinate functions $x^{i}$ on $\mathbb{R}^{p \mid q}$. Consequently, we must have 
$\partial_{t} c^{i}=c^{\sharp}\left(a_{i}\right)$. Equivalently, we have

$$
\begin{aligned}
\sum \frac{d c_{J}^{i}}{d t}(t, x) \theta^{J} & =a_{i}(c(t, x, \theta)) \\
& =a_{i}\left(\sum_{J} c_{J}(t, x) \theta^{J}\right) \\
& =a_{i}\left(c_{0}(t, x)+\sum_{J \neq 0} c_{J}(t, x) \theta^{J}\right) \\
& =a_{i}\left(c_{0}(t, x)\right)+\sum \frac{\partial a_{i}}{\partial x^{j}}\left(c_{0}(t, x)\right) c_{J}^{j}(t, x) \theta^{J}+\cdots \\
& =a_{i}\left(c_{0}(t, x)\right)+\sum f_{J}^{i}\left(\frac{\partial^{L} a_{i}}{\partial x^{L}}\left(c_{0}(t, x)\right), c_{K}(t, x)\right) \theta^{J}
\end{aligned}
$$

where $f_{J}^{i}$ are polynomial functions on some large euclidean space, $|L| \leq p$, and $|K| \leq q$. The fourth equality comes from the Taylor expansion for the function $a_{i}$ around $c_{0}(t, x)$. Equating the coefficients of the above relation, we obtain the system

$$
\begin{array}{ll}
\frac{d c_{0}^{i}}{d t}(t, x)=a_{i}\left(c_{0}(t, x)\right) & \text { for } i=1, \ldots, p, \\
\frac{d c_{J}^{i}}{d t}(t, x)=\sum f_{J}^{i}\left(\frac{\partial^{L} a_{i}}{\partial x^{L}}\left(c_{0}(t, x)\right), c_{K}(t, x)\right) & \text { for } 0 \neq|J| \leq q \\
& \text { and } i=1, \ldots, p+q .
\end{array}
$$

We solve first the system of the first $p$ equations to determine $c_{0}$, and then the first order system of differential equations determined by the last $(p+q)\left(2^{q}-1\right)$ equations. The initial condition of the system is given by the relations

$$
x^{i}=\sum c_{J}^{i}(0, x) \theta^{J} \quad \text { for } i=1, \ldots, p+q,
$$

which reflect the second condition in the statement of the lemma. By the general theory of systems of differential equations, the above system admits a unique solution. The lemma follows.

More generally, given an even vector field $X$ on a supermanifold $M$, and a parametrizing supermanifold $S$, we have a unique solution $\alpha: \mathbb{R} \times S \rightarrow M$ of the system

$$
\partial_{t} \circ \alpha^{\sharp}=\alpha^{\sharp} \circ X \quad \text { and }\left.\quad \alpha\right|_{0 \times S}=f
$$

for some initial condition $f: S \rightarrow M$. It is given by $\alpha=c \circ(1 \times f)$, where $c$ is the flow determined by $X$. The map $\alpha$ gives us a family of integral curves, parametrized by $S$, for the vector field $X$.

Next, we consider the odd case.

Lemma 2.4. Let $M$ be a compact supermanifold and $X$ be an odd vector field on $M$. Then there exists a unique map $\alpha: \mathbb{R}^{1 \mid 1} \times S \rightarrow M$ satisfying the two conditions

$$
D \circ \alpha^{\sharp}=\alpha^{\sharp} \circ X \quad \text { and }\left.\quad \alpha\right|_{0 \times S}=f
$$


for some initial condition $f: S \rightarrow M$. Here $D=\partial_{\theta}+\theta \partial_{t}$ is as usual.

Proof. Again, it is enough to solve the problem locally, for which we can assume that $M=\mathbb{R}^{p \mid q}$. Write $X=\sum a_{i} \partial_{x^{i}}$. Then the first relation on arbitrary functions $g$ on $\mathbb{R}^{p \mid q}$ gives

$$
\sum\left(\frac{\partial g}{\partial x^{i}} \circ \alpha\right) \frac{\partial \alpha^{i}}{\partial \theta}+\sum \theta\left(\frac{\partial g}{\partial x^{i}} \circ \alpha\right) \frac{\partial \alpha^{i}}{\partial t}=\sum\left(a_{i} \frac{\partial g}{\partial x^{i}}\right) \circ \alpha .
$$

Let us write $\alpha=G+\theta H$ with $G, H \in \mathscr{C}^{\infty}(I \times S)$ for some $I$ a neighborhood of 0 . Then, by Taylor's expansion, we have

$$
a_{i}(\alpha)=a_{i}(G)+\sum_{j} \theta \frac{\partial a_{i}}{\partial x^{j}}(G) H^{j}
$$

and (1) becomes

$$
H^{i}+\theta\left(\frac{\partial G^{i}}{\partial t}+\theta \frac{\partial H^{i}}{\partial t}\right)=a_{i}(G)+\sum_{j} \theta \frac{\partial a_{i}}{\partial x^{j}}(G) H^{j} .
$$

This is equivalent to the system

$$
a_{i}(G)=H^{i} \quad \text { and } \quad \frac{\partial G^{i}}{\partial t}(s, t)=\sum_{j} \frac{\partial a_{i}}{\partial x^{j}}(G(s, t)) H^{j}(s, t),
$$

which gives rise to the system

$$
\frac{\partial G^{i}}{\partial t}(s, t)=\sum_{j} \frac{\partial a_{i}}{\partial x^{j}}(G(s, t)) a_{j}(G(s, t)) .
$$

Now, $\sum_{j}\left(\partial a / \partial x^{j}\right) a_{j}$ is an even vector field on $\mathbb{R}^{p \mid q}$, so, by the previous lemma and the ensuing remark, the system (2) admits a unique solution once we know $G(0, s)$, which is given by the initial condition $f: S \rightarrow M$.

Remark 2.5. The flow of an odd (even) vector field defines actually an $\mathbb{R}^{1 \mid 1}$-action (respectively an $\mathbb{R}$-action) on a (compact) supermanifold.

Let $X$ be an odd vector field on a supermanifold $M$, and let $\alpha: \mathbb{R}^{1 \mid 1} \times M \rightarrow M$ be the flow of $X$. By definition, the following diagram is commutative:

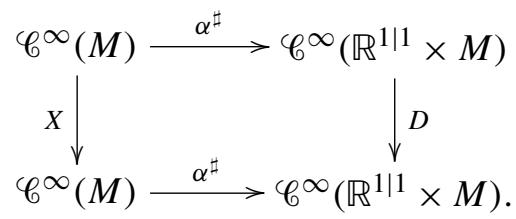

Let $u: S \rightarrow \mathbb{R}^{1 \mid 1} \times M$ be an $S$-point of $\mathbb{R}^{1 \mid 1} \times M$. Then $u^{\sharp} \circ D \circ \alpha^{\sharp}=u^{\sharp} \circ \alpha^{\sharp} \circ X$, which is to say that $\alpha_{* u}\left(D_{u}\right)=X_{\alpha(u)}$, where $\alpha_{*}$ is the differential of $\alpha$. If we denote $u=(t, \theta, x)$, then the last equation can also be written $\partial_{D} \alpha(t, \theta, x)=X(\alpha(t, \theta, x))$. This relation probably justifies our way of looking at a differential equation as a commutative diagram. See also [Šander 1980]. 
Again, let $X$ be an odd vector field on a supermanifold $M$. By the lemma above, $X$ defines a flow $\alpha: \mathbb{R}^{1 \mid 1} \times M \rightarrow M$. Define the map $\alpha_{0}: \mathbb{R} \times M \rightarrow M$ by $\alpha_{0}=\alpha \circ\left(i \times 1_{M}\right)$, where $i: \mathbb{R} \rightarrow \mathbb{R}^{1 \mid 1}$ is the standard inclusion map; $i$ is a group homomorphism if $\mathbb{R}$ and $\mathbb{R}^{1 / 1}$ are endowed with the standard group structures. Therefore $\alpha_{0}$ defines a flow map.

Lemma 2.6. The map $\alpha_{0}$ is the flow of the even vector field $X^{2}$.

Proof. Indeed, by definition $\alpha^{\sharp} \circ X=D \circ \alpha^{\sharp}$. Therefore

$$
\alpha^{\sharp} \circ X^{2}=D \circ \alpha^{\sharp} \circ X=D \circ D \circ \alpha^{\sharp}=\partial_{t} \circ \alpha^{\sharp} .
$$

Since $\partial_{t}$ commutes with $i^{\sharp} \otimes 1$, the claim follows.

Example. Let $D$ be the usual vector field on $\mathbb{R}^{1 / 1}$. Then the flow of $D$ is given by the group multiplication map $m: \mathbb{R}^{1 \mid 1} \times \mathbb{R}^{1 \mid 1} \rightarrow \mathbb{R}^{1 \mid 1}$. To see this, we should verify that $m$ fits into the diagram

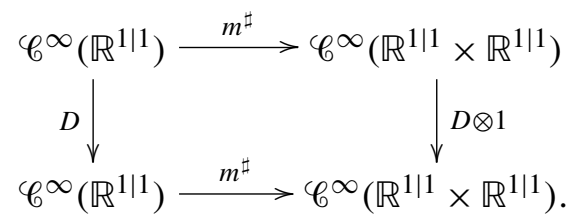

This is indeed the case: the diagram expresses that $D$ is a right invariant vector field.

\section{Connections on supermanifolds and their parallel transport}

The section describes the parallel transport along superpaths of a connection on a super vector bundle over a supermanifold. This follows closely the geometric idea of parallel transport associated to a connection on a vector bundle over a manifold.

3.1. Setup. Let $E$ be a super vector bundle over a supermanifold $M$, and let $\nabla$ be a connection on $E$ (see [Deligne and Morgan 1999]), that is, $\nabla: \Gamma(M, E) \rightarrow$ $\Omega^{1}(M, E)$ such that

$$
\nabla(f s)=d f \otimes s+f \nabla s \quad \text { for } f \in \mathscr{C}^{\infty}(M) \text { and } s \in \Gamma(M, E) .
$$

In particular, for $X \in \mathscr{X}(M)$ a vector field on $M$, we have $\nabla_{X}: \Gamma(M, E) \rightarrow \Gamma(M, E)$ with

$$
\nabla_{X}(f s)=X(f) s+(-1)^{p(X) p(f)} f \nabla_{X} s .
$$

Let $c: S \times \mathbb{R}^{1 \mid 1} \rightarrow M$ be a (family of) supercurve(s parametrized by a supermanifold $S$ ) in $M$. Consider the pullback connection $c^{*} \nabla$ and the derivation $\left(c^{*} \nabla\right)_{D}$ : $\Gamma\left(c^{*} E\right) \rightarrow \Gamma\left(c^{*} E\right)$. Here $D$ is the vector field $\partial_{\theta}+\theta \partial_{t}$ on $\mathbb{R}^{1 \mid 1}$, extended trivially to $S \times \mathbb{R}^{1 \mid 1}$. An element of $\Gamma\left(c^{*} E\right)$ is called a section of $E$ along $c$. We say that 
the section $s$ along $c$ is parallel if $\left(c^{*} \nabla\right)_{D} s=0$. In local coordinates, we can think of this as being a half-order differential equation. There are two reasons for that: first, the vector field $D$ squares to the vector field $d / d t$; second, for $2 n$ unknown functions we need $n$ values as initial data.

Proposition 3.1. Let $c: S \times \mathbb{R}^{1 \mid 1} \rightarrow M$ be a supercurve in the compact supermanifold $M$ (that is, the reduced manifold is compact). Let $\psi_{0} \in \Gamma\left(c_{0,0}^{*} E\right)$ be a section of $E$ along $c_{0,0}: S \rightarrow S \times \mathbb{R}^{1 \mid 1} \rightarrow M$, with the first map the standard inclusion $i_{0,0}: S \rightarrow S \times \mathbb{R}^{1 \mid 1}$. Then there exists a unique parallel section $\psi$ of $E$ along $c$ such that $\psi(0,0)=\psi_{0}$.

Proof. The fact that $\psi$ extends to all of $S \times \mathbb{R}^{1 \mid 1}$ is a standard argument on the flows of vector fields on compact manifolds. The existence (and uniqueness) of $\psi$ is then a local problem. Let $U \subseteq M$ be a trivializing neighborhood such that $E_{\mid U} \cong U \times \mathbb{R}^{p \mid q}(p \mid q$ is the rank of the bundle $E)$. Then the connection can be written as $\nabla=d+A$, for some $A \in \Omega^{1}(M) \otimes \operatorname{End}\left(\mathbb{R}^{p \mid q}\right)^{e v}$. The equation $\left(c^{*} \nabla\right)_{D} s=0$ with the given initial condition is then equivalent to the system

$$
\frac{\partial \psi}{\partial D}(s, t, \theta)+A(s, t, \theta) \psi(s, t, \theta)=0 \quad \text { and } \quad \psi(s, 0,0)=\psi_{0}(s),
$$

where $\psi$ is defined in a neighborhood of $S \hookrightarrow S \times \mathbb{R}^{1 \mid 1}$ with values in $\mathbb{R}^{p \mid q}$, and $A: S \times \mathbb{R}^{1 \mid 1} \rightarrow \operatorname{End}\left(\mathbb{R}^{p \mid q}\right)$ is short for $\left(c^{*} A\right)(D)$. If we write

$$
\begin{aligned}
& \psi(s, t, \theta)=\left(a^{i}(s, t)+\theta b^{i}(s, t)\right)_{i=1, \ldots, p+q}, \\
& A(s, t, \theta)=\left(c^{i j}(s, t)+\theta d^{i j}(s, t)\right)_{i, j=1, \ldots, p+q},
\end{aligned}
$$

then the system is equivalent to

$$
\begin{aligned}
b^{i}(s, t) & =-c^{i j}(s, t) a^{j}(s, t), \\
\frac{d a^{i}}{d t}(s, t) & =-\varepsilon\left(c^{i j}(s, t)\right) b^{j}(s, t)-d^{i j}(s, t) a^{j}(s, t), \\
a^{i}(s, 0) & =\psi_{0}^{i}(s) .
\end{aligned}
$$

Here $\varepsilon(a)=a$ if $a$ is even, and $\varepsilon(a)=-a$ if $a$ is odd. It is clear that this system admits a unique solution around $S \times(0,0)$. The proposition is proved.

Lemma 3.2 (Naturality in $S$ ). Let $c: S \times \mathbb{R}^{1 \mid 1} \rightarrow M$ be a supercurve in $M$, and let $\varphi: S^{\prime} \rightarrow S$ be an arbitrary map. Consider the supercurve $c^{\prime}: S^{\prime} \times \mathbb{R}^{1 \mid 1} \rightarrow M$ defined by $c^{\prime}=c \circ \bar{\varphi}$, where $\bar{\varphi}=\varphi \times 1_{\mathbb{R}^{1 \mid 1}}$. If $\psi$ is a parallel section along $c$, then $\psi \circ \bar{\varphi}$ is parallel along $c \circ \bar{\varphi}$.

Proof. $\psi$ is parallel along $c$ if $\left(c^{*} \nabla\right)_{D} \psi=0$. Observe that $\bar{\varphi}_{*} D=D$. Therefore

$$
\left(\bar{\varphi}^{*} c^{*} \nabla\right)_{D}\left(\bar{\varphi}^{*} \psi\right)=\bar{\varphi}^{\sharp}\left(\left(c^{*} \nabla\right)_{\bar{\varphi}_{*} D} \psi\right)=\bar{\varphi}^{\sharp}\left(\left(c^{*} \nabla\right)_{D} \psi\right)=0,
$$

since $\psi$ is parallel. That is, $\psi \bar{\varphi}$ is parallel along $c \bar{\varphi}$. 
Remark 3.3. We could just as well have defined a parallel section along a supercurve $c$ in $M$ to be a section $s$ along $c$ that satisfies the equation $\left(c^{*} \nabla\right)_{Q} s=0$, where $Q=\partial_{\theta}-\theta \partial_{t}$. Let us call such sections $Q$-parallel to distinguish them from the parallel sections defined above. Their relevance will become clear in property (iii) of Theorem 3.5.

3.2. Invariance under reparametrization. The usual parallel transport is invariant under reparametrization of paths. We will see in this subsection what that means in the super context.

Let $c: S \times \mathbb{R}^{1 \mid 1} \rightarrow M$ be a supercurve in $M$, and let $\psi$ be a parallel section of $E$ along $c$, that is, $\left(c^{*} \nabla\right)_{D} \psi=0$. Let $\varphi$ be a family, parametrized by $S$, of diffeomorphisms of $\mathbb{R}^{1 \mid 1}$ that preserve the distribution $\mathscr{D}$. In particular, $\varphi_{*} D=b D$, for some $b \in \mathscr{C}^{\infty}\left(S \times \mathbb{R}^{1 \mid 1}\right)$. Then we have

$$
\left((c \circ \varphi)^{*} \nabla\right)_{D}(\psi \circ \varphi)=\left(\left(c^{*} \nabla\right)_{\varphi_{*} D} \psi\right) \circ \varphi=b \cdot\left(\left(c^{*} \nabla\right)_{D} \psi\right) \circ \varphi=0 .
$$

Therefore, we conclude that if $\psi$ is a parallel section of $E$ along $c$, then $\psi \circ \varphi$ is a parallel section of $E$ along $c \circ \varphi$. We say that the parallel transport defined by the connection is invariant under reparametrization. (In our case, "reparametrization" refers to diffeomorphisms that preserve a distribution.)

This notion of parallel transport along superpaths generalizes the usual notion of parallel transport along paths associated with a connection in the sense that a parallel section in the old sense is parallel in the new sense, and the new parametrization invariance is compatible with the parametrization invariance in the old sense (a detailed discussion can be found in [Dumitrescu 2006, Section 4.3]).

3.3. Recovering the connection from the super parallel transport. We next ask, Given a connection on a super vector bundle and its associated parallel transport, how can we recover the connection? The answer goes as follows.

Giving a connection $\nabla$ on $E$ over $M$ amounts to specifying for each vector field $X$ on $M$ an $X$-derivation $\nabla_{X}=\tilde{X}: \Gamma(M, E) \rightarrow \Gamma(M, E)$, that is,

$$
\tilde{X}(f s)=X(f) s+(-1)^{p(X) p(f)} f \tilde{X}(s) \text { for } f \in \mathscr{C}^{\infty}(M) \text { and } s \in \Gamma(M, E),
$$

such that the correspondence $X \mapsto \tilde{X}$ is $\mathscr{C}^{\infty}(M)$-linear.

Let $X$ be an $o d d$ vector field on $M$, and let $\alpha=\alpha_{X}: \mathbb{R}^{1 \mid 1} \times M \rightarrow M$ be the flow of $X$. By definition (see Section 2.6), $X$ fits into the diagram

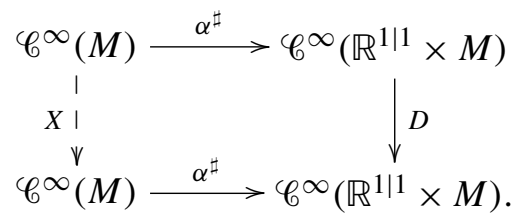


The pullback-connection via the path $\alpha$ will define, via the vector field $D$, a $D$-derivation $\widetilde{D}$ on the sections of the pullback bundle

$$
\Gamma\left(\mathbb{R}^{1 \mid 1} \times M, \alpha^{*} E\right)=\mathscr{C}^{\infty}\left(\mathbb{R}^{1 \mid 1} \times M\right) \otimes_{\mathscr{C}^{\infty}(M)} \Gamma(M, E) .
$$

Lemma 3.4. $\widetilde{D}=D \otimes 1+1 \otimes \tilde{X}$, where the right hand side is defined by

$$
f \otimes s \mapsto D f \otimes s+f \otimes \tilde{X} s \quad \text { for } f \in \mathscr{C}^{\infty}\left(\mathbb{R}^{1 \mid 1} \times M\right) \text { and } s \in \Gamma(M, E) .
$$

Proof. Indeed, both sides are $D$-derivations, and they coincide on sections of $E$ pulled back via the map $\alpha$. To see the latter, write $\alpha^{*} s=1 \otimes s$ for $s \in \Gamma(M, E)$. Then

$$
\widetilde{D}(1 \otimes s)=\left(\alpha^{*} \nabla\right)_{D}\left(\alpha^{*} s\right)=\alpha^{*}\left(\nabla_{X} s\right)=1 \otimes(\tilde{X} s)=(D \otimes 1+1 \otimes \tilde{X})(1 \otimes s) .
$$

Now, the parallel transport depicts in particular the parallel sections along $c$ in the direction of $D$. That information is enough to determine $\widetilde{D}: \Gamma\left(c^{*} E\right) \rightarrow$ $\Gamma\left(c^{*} E\right)$ as a $D$-derivation. Indeed, if $s_{i}$ for $i=1, \ldots, p+q$ locally are linearly independent parallel sections, then any $s \in \Gamma\left(c^{*} E\right)$ can be written $s=\sum f_{i} s_{i}$ with $f_{i} \in \mathscr{C}^{\infty}\left(\mathbb{R}^{1 \mid 1} \times M\right)$. Then $\widetilde{D}\left(\sum f_{i} s_{i}\right)=\sum D\left(f_{i}\right) s_{i}$. By the lemma above, we have in particular $\widetilde{D}\left(\alpha^{*} s\right)=\alpha^{*}(\tilde{X} s)$ for $s \in \Gamma(M, E)$, and since $\alpha^{*}: \Gamma(M, E) \rightarrow$ $\Gamma\left(\mathbb{R}^{1 \mid 1} \times M, \alpha^{*} E\right)$ is injective, knowing $\widetilde{D}$ uniquely determines $\tilde{X}$.

Let now $X$ be an even vector field on $M$, and let $\alpha: \mathbb{R} \times M \rightarrow M$ be the flow determined by $X$. Let $\hat{\alpha}: \mathbb{R}^{1 \mid 1} \times M \rightarrow M$ be the trivial extension of $\alpha$, that is, $\hat{\alpha}=\alpha \circ\left(p \times 1_{M}\right)$, where $p: \mathbb{R}^{1 \mid 1} \rightarrow \mathbb{R}$ is the usual projection (which on functions is the inclusion of functions on $\mathbb{R}$ into forms on $\mathbb{R}$ ). Then

$$
\left(\hat{\alpha}^{*} \nabla\right)_{D}\left(\hat{\alpha}^{*} s\right)=\theta\left(\alpha^{*} \nabla\right)_{\partial_{t}}\left(\alpha^{*} s\right)=\theta \alpha^{*}\left(\nabla_{X} s\right),
$$

for all sections $s \in \Gamma(M, E)$. Since, as before, $\alpha^{*}$ is injective, the lift of $D$ along $\alpha$ determines the lift of $X$ given by the connection.

In this way, via the super parallel transport, we can lift all the vector fields on $M$ to the derivations given by the connection; in other words, the super parallel transport recovers the connection.

3.4. Parallel transport along superpaths. Let $(t, \theta) \in \mathbb{R}_{+}^{1 \mid 1}(S)$ be an $S$-point of $\mathbb{R}_{+}^{1 \mid 1}$. We define a super analogue of the interval $I_{t}=[0, t]$ as follows:

Consider the triplet

$$
S \underset{i_{(0,0)}}{\longrightarrow} S \times \mathbb{R}^{1 \mid 1} \underset{i_{(t, \theta)}}{\longrightarrow} S
$$

with $i_{(0,0)}(s)=(s, 0,0)$ and $i_{(t, \theta)}(s)=(s, t(s), \theta(s))$. Here $\mathbb{R}^{1 \mid 1}$ is endowed with the standard metric structure given by the odd vector field $D=\partial_{\eta}+\eta \partial_{u}$ in coordinates $(u, \eta)$ on $\mathbb{R}^{1 \mid 1}$ (see Section 2.3). We denote this (family of) superinterval(s) by $I_{(t, \theta)}$. 
Let $x$ and $y$ be $S$-points of $M$. A superpath in $M$ parametrized by the superinterval $I_{(t, \theta)}$ and with endpoints $x$ and $y$ is an equivalence class of supercurves $c: S \times \mathbb{R}^{1 \mid 1} \rightarrow M$ with $c \circ i_{0,0}=c(0,0)=x$ and $c \circ i_{t, \theta}=c(t, \theta)=y$, respectively, such that $c \sim c^{\prime}$ if there exists an $\varepsilon>0$ such that $c(u, \eta)=c^{\prime}(u, \eta)$ for all $(-\eta, 0)<(u, \eta)<(t+\eta, \theta)$. Here, " $<$ " is a partially defined order as follows: for $(t, \theta),(u, \eta) \in \mathbb{R}^{1 \mid 1}(S)$, we say

$$
(u, \eta)<(t, \theta) \quad \text { if }(t, \theta)(u, \eta)^{-1} \in \mathbb{R}_{+}^{1 \mid 1}(S) .
$$

Recall that $\mathbb{R}^{1 \mid 1}$ is a super Lie group (see Section 2.4) with the group structure

$$
(t, \theta),(s, \eta) \mapsto(t, \theta)(s, \eta):=(t+s+\theta \eta, \theta+\eta) .
$$

In particular, for any supermanifold $S, \mathbb{R}^{1 \mid 1}(S)=\mathbf{S M}\left(S, \mathbb{R}^{1 \mid 1}\right)$ is not just a set but a group. $\mathbb{R}_{+}^{1 \mid 1}$ is the open subsupermanifold in $\mathbb{R}^{1 \mid 1}$ whose reduced part is $\mathbb{R}_{+}=(0, \infty)$. For short, such a superpath is denoted $c: I_{(t, \theta)} \rightarrow M$.

Now let $c: I_{(t, \theta)} \rightarrow M$ be a superpath in $M$. Then the connection $\nabla$ on the bundle $E$ will determine a vector bundle homomorphism

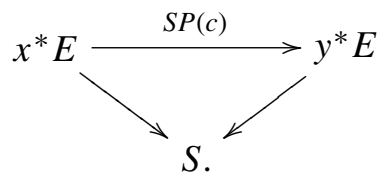

$S P(c)$ is a $\mathscr{C}^{\infty}(S)$-linear map $S P(c): \Gamma\left(S, x^{*} E\right) \rightarrow \Gamma\left(S, y^{*} E\right)$ described by the diagram

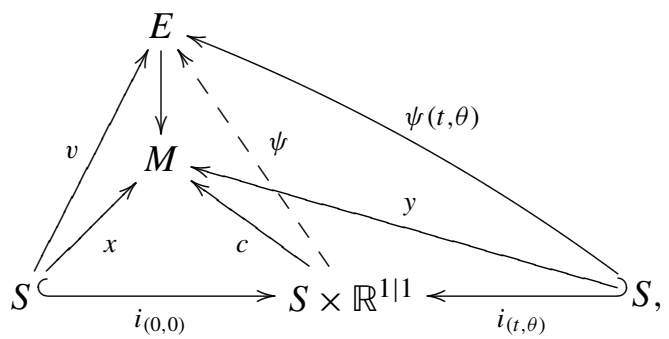

that is, $\operatorname{SP}(c)(v)=\psi(t, \theta)$, where $\psi$ is the unique parallel section of $E$ along the supercurve $c$ such that $\psi(0,0)=v$. Since the solution $\psi$ depends on the local data, it turns out that the map $S P(c)$ is well defined, that is, it does not depend on a representative for the superpath $c: I_{(t, \theta)} \rightarrow M$. It is clearly a $\mathscr{C}^{\infty}(S)$-linear map; therefore it defines a bundle map $S P(c): x^{*} E \rightarrow y^{*} E$.

The map $S P$ satisfies the usual properties of a parallel transport map, that is, it is compatible with gluing superpaths and is invariant under reparametrizations (that is, diffeomorphisms of superintervals that preserve the fiberwise conformal structure on $\mathbb{R}^{1 \mid 1}$ ). This forms the content of the following theorem: 
Theorem 3.5. Any connection $\nabla$ on a super vector bundle $E$ over a supermanifold $M$ gives rise to a correspondence $S P(\nabla)=S P$

$$
I_{t, \theta} \stackrel{c}{\longrightarrow} M \quad \stackrel{S P(\nabla)}{\longrightarrow} \quad c_{0,0}^{*} E \longrightarrow c_{t, \theta}^{*} E
$$

satisfying the following properties:

(i) The correspondence $c \mapsto S P(c)$ is smooth and natural in $S$ (see Lemma 3.2). Smoothness means that if $c$ is a family of smooth superpaths parametrized by a supermanifold $S$, then the map $S P(c): c_{0,0}^{*} E \rightarrow c_{t, \theta}^{*} E$ is a smooth bundle map over $S$.

(ii) (Compatibility under gluing.) If $c: I_{t, \theta} \rightarrow M$ and $c^{\prime}: I_{t^{\prime}, \theta^{\prime}} \rightarrow M$ are two superpaths in $M$ such that $c^{\prime} \equiv c \circ R_{t, \theta}$ on some neighborhood $S \times U$ of $S \times(0,0) \hookrightarrow S \times \mathbb{R}^{1 \mid 1}$, with $U$ an open subsupermanifold in $\mathbb{R}^{1 \mid 1}$ containing $(0,0)$, we have $S P\left(c^{\prime} \cdot c\right)=S P\left(c^{\prime}\right) \circ S P(c)$, where $c^{\prime} \cdot c: I_{t^{\prime}+t+\theta^{\prime} \theta, \theta^{\prime}+\theta} \rightarrow M$ is obtained from $c$ and $c^{\prime}$ by gluing them along their "common endpoint", that is,

$$
\left(c^{\prime} \cdot c\right)(s, u, \eta)= \begin{cases}c(s, u, \eta) & \text { if }(u, \eta)<(t+\varepsilon, \theta), \\ c^{\prime}\left(s,(u, \eta)(t, \theta)^{-1}\right) & \text { if }(t-\varepsilon, \theta)<(u, \eta) .\end{cases}
$$

(Here $R_{t, \theta}: S \times \mathbb{R}^{1 \mid 1} \rightarrow S \times \mathbb{R}^{1 \mid 1}$ is the right translation by $(t, \theta)$ in the $\mathbb{R}^{1 \mid 1}$ direction, that is, $R_{t, \theta}(s,(u, \eta))=(s,(u, \eta)(t, \theta))$.)

(iii) For any superpath $c: I_{t, \theta} \rightarrow M$, the bundle map $S P(c): c_{0,0}^{*} E \rightarrow c_{t, \theta}^{*} E$ is an isomorphism, with inverse given by $P S(\bar{c}): c_{t, \theta}^{*} E \rightarrow c_{0,0}^{*} E$, where $\bar{c}: I_{t, \theta} \rightarrow M$ is given by $\bar{c}(u, \eta)=c\left((u, \eta)^{-1}(t, \theta)\right)$ and, for a superpath $\alpha$ in $M, P S(\alpha)$ denotes $Q$-parallel transport along $\alpha$ (see Remark 3.3).

(iv) (Invariance under reparametrization.) Given $c: I_{t, \theta} \rightarrow M$ a superpath in $M$ and $\varphi: I_{s, \eta} \rightarrow I_{t, \theta}$ a family of diffeomorphisms of superintervals that preserve the vertical distribution, we have $\operatorname{SP}(c \circ \varphi)=S P(c)$.

Moreover, if $\nabla \neq \nabla^{\prime}$, then $\operatorname{SP}(\nabla) \neq \operatorname{SP}\left(\nabla^{\prime}\right)$.

Proof of (ii). Since the construction of parallel transport is natural in $S$ (see Lemma 3.2 ), it is enough to consider the case when $S$ is "small" and $c$ and $c^{\prime}$ map to a trivializing neighborhood $U \subseteq M$ for $E$ such that $\left.E\right|_{U} \cong U \times \mathbb{R}^{p \mid q}$ and $\nabla=d+A$. If $\psi$ is a superparallel section along $c$ with $\psi(0,0)=\psi_{0}$ and $\psi^{\prime}$ is parallel along $c^{\prime}$ with $\psi^{\prime}(0,0)=\psi(t, \theta)$, then $\psi^{\prime} \cdot \psi$ defined by

$$
\psi^{\prime} \cdot \psi(s, u, \eta)= \begin{cases}\psi(s, u, \eta) & \text { if }(u, \eta)<(t+\varepsilon, \theta), \\ \psi^{\prime}\left(s,(u, \eta)(t, \theta)^{-1}\right) & \text { if }(t-\varepsilon, \theta)<(u, \eta)\end{cases}
$$

is a parallel section along $c^{\prime} \cdot c$. (Observe that $\psi^{\prime} \cdot \psi$ is well defined by Proposition 3.1.) To show this, it is enough to prove the following lemma. 
Lemma 3.6. Let $c: S \times \mathbb{R}^{1 \mid 1} \rightarrow M$ be a superpath in $M$, and let $A \in \Omega^{1}(M) \otimes$ $\operatorname{End}\left(\mathbb{R}^{p \mid q}\right)^{e v}$. Also let $\psi: S \times \mathbb{R}^{1 \mid 1} \rightarrow \mathbb{R}^{p \mid q}$ be such that

$$
\partial_{D} \psi+\left(c^{*} A\right)(D) \psi=0 .
$$

If $\bar{c}=c \circ R_{(t, \theta)}$ and $\bar{\psi}=\psi \circ R_{(t, \theta)}$, then

$$
\partial_{D} \psi+\left(\bar{c}^{*} A\right)(D) \psi=0 .
$$

Proof. Let $R$ be short for $R_{(t, \theta)}$. Then $R^{\sharp}$ extends to $\mathbb{R}^{p \mid q}$-valued functions. Also, the vector field $D$ is invariant under right translations, that is, $R_{*} D=D$ or, written differently, $D \circ R^{\sharp}=R^{\sharp} \circ D$. Applied to the $\mathbb{R}^{p \mid q}$-valued function $\psi$, this gives

$$
\partial_{D}(\psi \circ R)=\left(\partial_{D} \psi\right) \circ R
$$

On the other hand,

$$
\left(\bar{c}^{*} A\right)(D)=\left(R^{*}\left(c^{*} A\right)\right)(D)=R^{\sharp}\left(c^{*} A\left(R_{*} D\right)\right)=R^{\sharp}\left(c^{*} A(D)\right)=\left(c^{*} A(D)\right) \circ R .
$$

Therefore we have

$$
\begin{aligned}
\partial_{D} \psi+\left(\bar{c}^{*} A\right)(D) \psi & =\partial_{D}(\psi \circ R)+R^{*}\left(c^{*} A\right)(D)(\psi \circ R) \\
& =\partial_{D}(\psi) \circ R+\left(\left(c^{*} A\right)(D) \psi\right) \circ R=0 .
\end{aligned}
$$

Proof of (iii). It is enough to assume that $c$ maps to a trivializing neighborhood, as before. Then $\psi$ is parallel along $c$ if $\partial_{D} \psi+\left(c^{*} A\right)(D) \psi=0$. Consider the section $\bar{\psi}$ along $\bar{c}$ defined by $\psi(s, u, \eta)=\psi\left(s,(u, \eta)^{-1}(t, \theta)\right)$. Then $\psi$ is $Q$-parallel along $\bar{c}$. To see this, it is enough to prove this lemma:

Lemma 3.7. Let $c: S \times \mathbb{R}^{1 \mid 1} \rightarrow M$ be a superpath in $M$, and let $A \in \Omega^{1}(M) \otimes$ $\operatorname{End}\left(\mathbb{R}^{p \mid q}\right)^{\text {ev }}$. Let also $\psi: S \times \mathbb{R}^{1 \mid 1} \rightarrow \mathbb{R}^{p \mid q}$ be such that

$$
\partial_{D} \psi+\left(c^{*} A\right)(D) \psi=0 .
$$

If $\bar{c}=c \circ R_{(t, \theta)} \circ I$ and $\bar{\psi}=\psi \circ R_{(t, \theta)} \circ I$, where $I: S \times \mathbb{R}^{1 \mid 1} \rightarrow S \times \mathbb{R}^{1 \mid 1}:(s, u, \eta) \mapsto$ $(s,-u,-\eta)$ is the inversion map, then $\partial_{Q} \psi+\left(\bar{c}^{*} A\right)(Q) \psi=0$.

Proof. Let us begin by showing that, via the inversion map $I: \mathbb{R}^{1 \mid 1} \rightarrow \mathbb{R}^{1 \mid 1}:(t, \theta) \mapsto$ $(-t,-\theta)$, we have $I_{*} D=-Q$. For that, we need to show the commutativity of

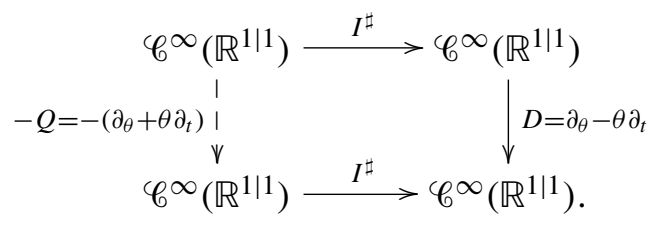


Following the diagram both ways, we have
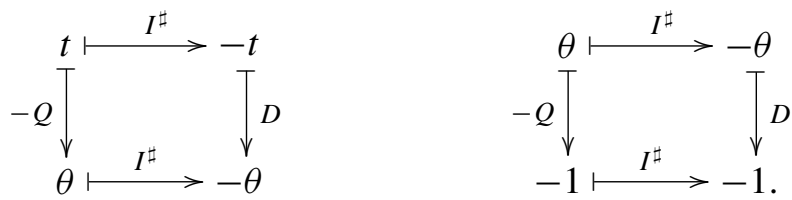

Returning to the proof of the lemma, let us notice that $\psi$ can be written $\psi=I^{\sharp} R^{\sharp} \psi$, where $R$ is short for $R_{(t, \theta)}$. Then

$$
\partial_{Q}(\Psi)=\partial_{Q}\left(I^{\sharp} R^{\sharp} \psi\right)=-I^{\sharp} \partial_{D} R^{\sharp} \psi=-I^{\sharp} R^{\sharp} \partial_{D} \psi,
$$

where the second equality is true by $\partial_{Q} I^{\sharp}=-I^{\sharp} \partial_{D}$ above and the third equality is true since $D$ is a right-invariant vector field, that is, $R^{\sharp} \partial_{D}=\partial_{D} R^{\sharp}$.

On the other side, we have

$$
\begin{aligned}
\left(\bar{c}^{*} A\right)(Q) & =\left(I^{*} R^{*} c^{*} A\right)(Q) \\
& =I^{\sharp}\left(R^{*}\left(c^{*} A\right)\left(I_{*}(Q)\right)\right)=-I^{\sharp}\left(R^{*}\left(c^{*} A\right)(D)\right) \\
& =-I^{\sharp} R^{\sharp}\left(\left(c^{*} A\right)(D)\right),
\end{aligned}
$$

where we used that the fact that

$$
\left(f^{*} \omega\right)(Y)=f^{\sharp}\left(\omega\left(f_{*} Y\right)\right),
$$

with $f: N \rightarrow M$ an arbitrary map of supermanifolds, $\omega \in \Omega^{1}(M)$, and $Y \in \mathscr{X}(N)$. (The relation is true provided $f_{*} Y$ exists, which is true in our cases.) Therefore

$$
\begin{aligned}
\partial_{Q} \psi+\left(\bar{c}^{*} A\right)(Q) \psi & =-I^{\sharp} R^{\sharp} \partial_{D} \psi-I^{\sharp} R^{\sharp}\left(\left(c^{*} A\right)(D)\right)\left(I^{\sharp} R^{\sharp} \psi\right) \\
& =-\left(\partial_{D} \psi+\left(c^{*} A\right)(D) \psi\right) \circ R \circ I=0 .
\end{aligned}
$$

The conclusion of (iii) follows.

3.5. The parallel transport of $(\nabla, A)$. In the end of this section we define a notion of A-parallel transport for the pair consisting of a connection and a bundle endomorphism $A$, and see that it converges (by an "inverse adiabatic limit" process) to the parallel transport of the connection. In particular, this means that the $A$-parallel transport is reparametrization invariant in the limit.

Let $E$ be a super vector bundle over a supermanifold $M$. Let $(\nabla, A)$ be a pair consisting of a (grading-preserving) connection $\nabla$ on $E$ and $A \in \Gamma(M$, End $E)$ an odd endomorphism of $E$. Let $c: S \times \mathbb{R}^{1 \mid 1} \rightarrow M$ in $M$ be a family of supercurves parametrized by a supermanifold $S$. A section $\psi \in \Gamma\left(c^{*} E\right)$ of $E$ along $c$ is $A$ parallel if it satisfies the equation

$$
\left(c^{*} \nabla\right)_{D} \psi-\left(c^{*} A\right) \psi=0 .
$$


This is again a "half-order" differential equation. In local coordinates, if $E_{\mid U} \cong$ $U \times \mathbb{R}^{p \mid q}$, then $\nabla=d+a$ with $a \in \Omega^{1}(M \text {, End } E)^{\text {odd }}$, and Equation (3) can be written

$$
\partial_{D} \psi+\left(c^{*} a\right)(D) \psi-\left(c^{*} A\right) \psi=0,
$$

where $D=\partial_{\eta}+\eta \partial_{u}$. Suppose for simplicity that $(u, \eta)$ runs on the superinterval $I_{(T, \tau)}$ for $(T, \tau) \in \mathbb{R}_{+}^{1 \mid 1}(S)$ an $S$-superpoint of $\mathbb{R}_{+}^{1 \mid 1}$. Recall that $I_{(T, \tau)}$ is defined by the embeddings

$$
S \underset{i_{(0,0)}}{\longrightarrow} S \times \mathbb{R}^{1 \mid 1} \underset{i_{(T, \tau)}}{\longrightarrow} S .
$$

For $\lambda>0$, let

$$
\varphi_{\lambda}: I_{(\lambda T, \sqrt{\lambda} \tau)} \rightarrow I_{(T, \tau)}:(t, \theta) \mapsto\left(\frac{1}{\lambda} t, \frac{1}{\sqrt{\lambda}} \theta\right)
$$

be the "rescaling" diffeomorphism that preserves the distribution $\mathscr{D}$. Then $\widetilde{\psi}$ is $A$-parallel with respect to $\tilde{c}=c \circ \varphi_{\lambda}$ if

$$
\partial_{\tilde{D}} \widetilde{\psi}+\left(c^{*} a\right)(\widetilde{D}) \widetilde{\psi}-\left(\tilde{c}^{*} A\right) \widetilde{\psi}=0,
$$

where $\widetilde{D}=\partial_{\theta}+\theta \partial_{t}$. If we write $\widetilde{\psi}=\psi^{\lambda} \circ \varphi_{\lambda}=\varphi_{\lambda}^{\sharp}\left(\psi^{\lambda}\right)$ then the last equation can be rewritten

$$
\partial_{\widetilde{D}}\left(\varphi_{\lambda}^{\sharp}\left(\psi^{\lambda}\right)\right)+\varphi_{\lambda}^{*}\left(c^{*} a\right)(\widetilde{D}) \varphi_{\lambda}^{\sharp}\left(\psi^{\lambda}\right)-\varphi_{\lambda}^{\sharp}\left(c^{*} A\right) \varphi_{\lambda}^{\sharp}\left(\psi^{\lambda}\right)=0,
$$

An easy calculation shows that $\varphi_{\lambda *}(\widetilde{D})=(1 / \sqrt{\lambda}) D$, which can be rewritten as $\partial_{\widetilde{D}} \varphi_{\lambda}^{\sharp}=(1 / \sqrt{\lambda}) \varphi_{\lambda}^{\sharp} \partial_{D}$, and the last equation is equivalent to

$$
\frac{1}{\sqrt{\lambda}} \varphi_{\lambda}^{\sharp} \partial_{D} \psi^{\lambda}+\frac{1}{\sqrt{\lambda}} \varphi_{\lambda}^{\sharp}\left(\left(c^{*} a\right)(D)\right) \varphi_{\lambda}^{\sharp}\left(\psi^{\lambda}\right)-\varphi_{\lambda}^{\sharp}\left(\left(c^{*} A\right) \psi^{\lambda}\right)=0 ;
$$

therefore $\partial_{D} \psi^{\lambda}+\left(c^{*} a\right)(D) \psi^{\lambda}-\sqrt{\lambda}\left(c^{*} A\right) \psi^{\lambda}=0$.

If we let $\lambda \rightarrow 0$ we see that $\psi^{\lambda} \longrightarrow \psi^{0}$, where $\psi^{0}$ is the parallel section along $c$ determined by the connection $\nabla$. We conclude that the parallel transport defined by $(\nabla, A)$ converges in the "inverse adiabatic limit" to the parallel transport of $\nabla$, which is in particular invariant under reparametrization. Symbolically we write

$$
S P(\nabla, A) \longrightarrow S P(\nabla) .
$$

\section{Superconnections and parallel transport}

In this section we prove our main result: Any superconnection $\mathbb{A}$ on a $\mathbb{Z} / 2$-graded vector bundle over a manifold gives rise to a parallel transport $S P(\mathbb{A})$ which converges to the parallel transport $\operatorname{SP}\left(\mathbb{A}_{1}\right)$ determined by $\mathbb{A}_{1}$, the connection part of the superconnection. 
4.1. Preliminaries. Start with a $\mathbb{Z} / 2$-graded vector bundle $E$ over a manifold $M$, and consider a grading-preserving connection $\nabla$ on $E$, together with an End $E$ valued form $A$ on $M, A \in\left(\Omega^{*}(M, \text { End } E)\right)^{\text {odd }}$. Combining these two pieces, we obtain a Quillen connection $\mathbb{A}=\nabla+A$ on $E$.

Recall the identification in Section 2.5.

$$
\underline{\mathbf{S M}}\left(\mathbb{R}^{1 \mid 1}, M\right)=\underline{\mathbf{S M}}(\mathbb{R}, \Pi T M),
$$

which for a supermanifold $S$ gives

$$
\mathbf{S M}\left(S \times \mathbb{R}^{1 \mid 1}, M\right) \cong \mathbf{S M}(S \times \mathbb{R}, \Pi T M) .
$$

Let $c: S \times \mathbb{R}^{1 \mid 1} \rightarrow M$ be a supercurve in $M$. Lift it to a supercurve $\tilde{c}$ in $\Pi T M$ as follows:

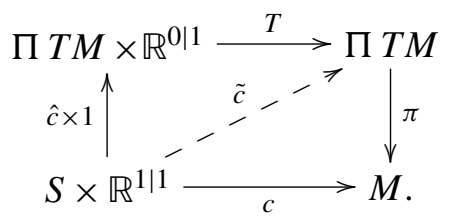

In other words, $\tilde{c}=T \circ(\hat{c} \times 1)$, where $T$ is the $\mathbb{R}^{0 \mid 1}$ action map on $\Pi T M$ (see Lemma 2.2). The map $\hat{c}: S \times \mathbb{R} \rightarrow \Pi T M$ corresponds to $c: S \times \mathbb{R}^{1 \mid 1} \rightarrow M$ under the above identification. The map $\pi$ is given on functions by $\pi^{\sharp}: \mathscr{C}^{\infty}(M) \rightarrow \mathscr{C}^{\infty}(\Pi T M)=$ $\Omega(M)$, the inclusion of functions on $M$ into the space of differential forms on $M$. Claim. The above diagram is commutative.

Proof of Claim. It is enough to show that

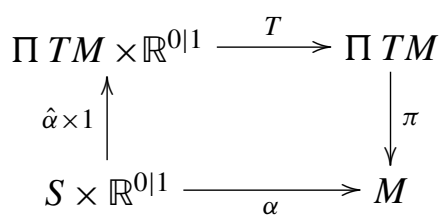

is commutative for $S$ an arbitrary supermanifold, and $\alpha: S \times \mathbb{R}^{0 \mid 1} \rightarrow M$ an arbitrary map. Here $\hat{\alpha}: S \rightarrow \Pi T M$ corresponds to $\alpha$ via Lemma 2.1.

This translates into the commutativity of

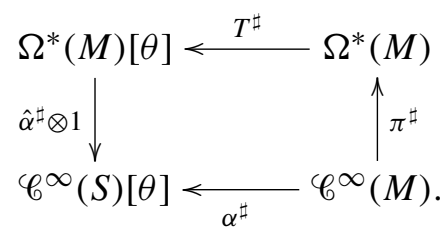

Recall from the proof of Lemma 2.1 that if $\alpha^{\sharp}: f \mapsto a^{\sharp}(f)+\theta X_{a}(f)$ for $a \in M(S)$ and $X_{a} \in T M_{a}$, then $\hat{\alpha}^{\sharp}: \Omega^{*}(M) \rightarrow \mathscr{C}^{\infty}(S)[\theta]$ is determined by requiring that 
$f \mapsto a^{\sharp}(f)$ and $d f \mapsto X_{a}(f)$. Therefore we have

$$
f \stackrel{\pi^{\sharp}}{\longmapsto} f \stackrel{T^{\sharp}}{\longmapsto} f+\theta d f \stackrel{\hat{\alpha}^{\sharp} \otimes 1}{\longmapsto} a^{\sharp}(f)+\theta X_{a}(f)=\alpha^{\sharp}(f) .
$$

To complete the proof of the claim, it is enough to replace $S \mapsto S \times \mathbb{R}$ and $\alpha \mapsto c$ in the above considerations.

Remark 4.1. It is not hard to check that the construction $c \mapsto \tilde{c}$ is natural in $S$, that is,

$$
c \widetilde{(\varphi \times 1)}=\tilde{c} \circ(\varphi \times 1)
$$

for $\varphi: S^{\prime} \rightarrow S$ an arbitrary map of supermanifolds.

Given the supercurve $c$, consider the pullback diagram

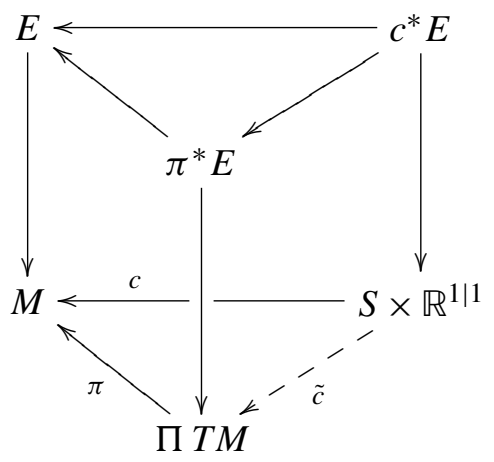

where $\tilde{c}$ is as above. We say a section $\psi \in \Gamma\left(c^{*} E\right)$ of $E$ along $c$ is $\mathbb{A}$-parallel if it satisfies the equation

$$
\left(c^{*} \nabla\right)_{D} \psi-\left(\tilde{c}^{*} A\right) \psi=0 .
$$

This is again a "half-order" differential equation. It is equivalent to the equation

$$
\left(\tilde{c}^{*}\left(\pi^{*} \nabla\right)\right)_{D} \psi-\left(\tilde{c}^{*} A\right) \psi=0 .
$$

Therefore $\psi \in \Gamma\left(c^{*} E\right)$ is $\mathbb{A}$-parallel if and only if $\psi$ is $A$-parallel along the lift $\tilde{c}$ with respect to the pair $\left(\pi^{*} \nabla, A \in \Gamma\left(\operatorname{End}\left(\pi^{*} E\right)\right)\right)$ on the bundle $\pi^{*} E \rightarrow \Pi T M$, as defined in Section 3.5. Therefore Proposition 3.1 gives the following:

Proposition 4.2. Let $c: S \times \mathbb{R}^{1 \mid 1} \rightarrow M$ be a supercurve in the compact manifold $M$. Let $\psi_{0} \in \Gamma\left(c_{0,0}^{*} E\right)$ be a section of $E$ along $c_{0,0}: S \rightarrow M$. Then there exists a unique A-parallel section $\psi$ of E along c such that $\psi(0,0)=\psi_{0}$.

4.2. Parallel transport along superpaths. Suppose $c: I_{t, \theta} \rightarrow M$ is a superpath in $M$ with $c(0,0)=x$ and $c(t, \theta)=y$. Then the A-parallel transport of $\nabla$, and $A \in \Omega^{*}(M$, End $E)$ will determine a bundle homomorphism $S P(c): x^{*} E \rightarrow y^{*} E$. 
This is defined as in Section 3.4 by a $\mathscr{C}^{\infty}(S)$-linear map $S P(c): \Gamma\left(S, x^{*} E\right) \rightarrow$ $\Gamma\left(S, y^{*} E\right)$ described by the diagram

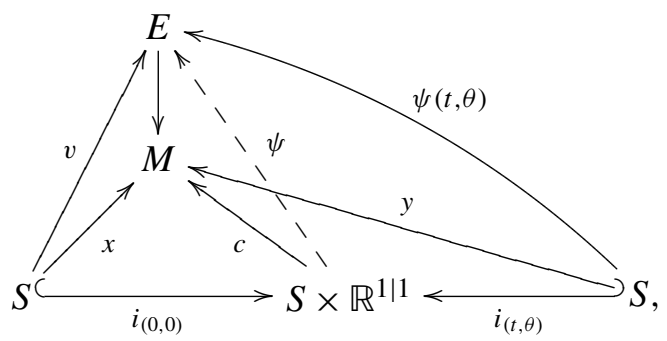

that is, $\operatorname{SP}(c)(v)=\psi(t, \theta)$, where $\psi$ is the unique $\mathbb{A}$-parallel section with respect to the pair $(\nabla, A)$ of $E$ along the supercurve $c$ such that $\psi \circ i_{(0,0)}=v$.

4.3. Main theorem. We are now in the position to state our main theorem.

Theorem 4.3. Let $E$ be a $\mathbb{Z} / 2$-graded vector bundle over a manifold $M$. Let $\nabla$ be a grading preserving connection on $E$ and $A \in \Omega^{*}(M \text {, End } E)^{\text {odd }}$. The pair $(\nabla, A)$ gives rise to a correspondence $S P=S P(\nabla, A)$

$$
I_{t, \theta} \stackrel{c}{\longrightarrow} M \quad \longmapsto \quad S P \longrightarrow c_{0,0}^{*} E \longrightarrow c_{t, \theta}^{*} E
$$

such that

(i) the correspondence $c \mapsto S P(c)$ is smooth and natural in $S$ (see Lemma 3.2);

(ii) (Compatibility under gluing.) If $c: I_{t, \theta} \rightarrow M$ and $c^{\prime}: I_{t^{\prime}, \theta^{\prime}} \rightarrow M$ are two superpaths in $M$ such that $c^{\prime} \equiv c \circ R_{t, \theta}$ on some neighborhood $S \times U$ of $S \times(0,0) \hookrightarrow S \times \mathbb{R}^{1 \mid 1}$, with $U$ an open subsupermanifold in $\mathbb{R}^{1 \mid 1}$ containing $(0,0)$, we have

$$
S P\left(c^{\prime} \cdot c\right)=S P\left(c^{\prime}\right) \circ S P(c),
$$

where $c^{\prime} \cdot c: I_{t^{\prime}+t+\theta^{\prime} \theta, \theta^{\prime}+\theta} \rightarrow M$ is obtained from $c$ and $c^{\prime}$ by gluing them along their "common endpoint".

Moreover, if $\nabla \neq \nabla^{\prime}$ or $A \neq A^{\prime}$ then $\operatorname{SP}(\nabla, A) \neq \operatorname{SP}\left(\nabla^{\prime}, A^{\prime}\right)$. Also, $\operatorname{SP}(\nabla, A)$ converges in the inverse adiabatic limit to $\operatorname{SP}(\nabla)$.

Proof. The properties (i) and (ii) are clear from the construction of the parallel transport of the pair $(\nabla, A)$. Two different such pairs (superconnections) give rise to two different parallel transports, since the parallel transport recovers the superconnection, as we will show in Section 4.4. The inverse adiabatic limit process is described in Section 3.5.

Corollary 4.4 (The parallel transport of a superconnection). A superconnection $\mathbb{A}$ on the bundle $E$ over $M$ (in the sense of Quillen) gives rise in a unique way to 
$a$ (super)parallel transport based on $M$; namely, consider the parallel transport $\operatorname{SP}(\nabla, A)$ associated to the pair $\left(\nabla=\mathbb{A}_{1}, A=\sum_{i \neq 1} \mathbb{A}_{i}\right)$.

4.4. Recovering the superconnection. In this section we show how to recover a superconnection, that is, a pair $(\nabla, A)$, from the parallel transport associated to it. We have already seen in Section 3.5 how the parallel transport of $(\nabla, A)$ converges via an inverse adiabatic limit process to the parallel transport of $\nabla$, which further recovers the connection $\nabla$. See Section 3.3. We are only left to obtain $A \in \Omega^{*}(M$, End $E)$. To do that, let us consider the diagram

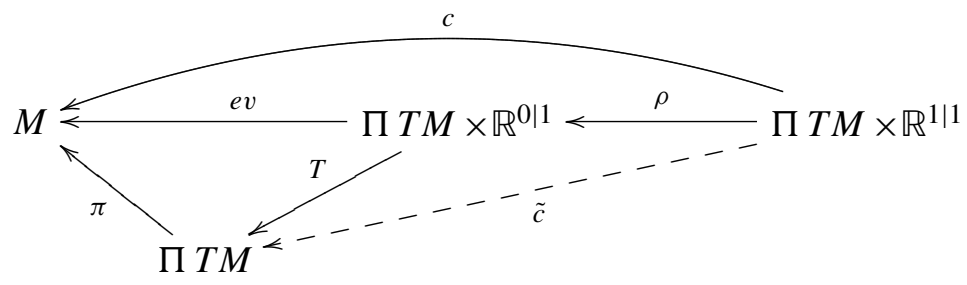

where $e v$ is the "evaluation" map as in the previous section and $\rho=1_{\Pi T M} \times p$, with $p: \mathbb{R}^{1 \mid 1} \rightarrow \mathbb{R}^{0 \mid 1}$ the natural projection map. Let us remark first that the lift of the curve $c=e v \circ \rho$ is the composition $T \circ \rho$. This holds by the naturality of lifts of supercurves (see Remark 4.1), and the fact that the lift of the "curve" $e v$ is given by $T$ (in diagram (4), if $\alpha=e v$ then $\widehat{e v}=1_{\Pi T M}$ ).

By definition, a section $\psi \in \Gamma\left(c^{*} E\right)$ of $E$ along $c$ is $\mathbb{A}$-parallel if

$$
\left(c^{*} \nabla\right)_{D} \psi-\left(\rho^{*} T^{*} A\right) \psi=0 .
$$

We therefore know the operator

$$
\left(c^{*} \nabla\right)_{D}-\rho^{*} T^{*} A: \Gamma\left(c^{*} E\right) \rightarrow \Gamma\left(c^{*} E\right)
$$

on parallel sections. But that is enough to determine it, since the parallel sections generate $\Gamma\left(c^{*} E\right)$ as a $\mathscr{C}^{\infty}\left(\Pi T M \times \mathbb{R}^{1 \mid 1}\right)$-module. On the other hand, we know the operator $\left(c^{*} \nabla\right)_{D}: \Gamma\left(c^{*} E\right) \rightarrow \Gamma\left(c^{*} E\right)$ since we know the connection $\nabla$. In this manner we determine the linear map $\rho^{*} T^{*} A$. Since both $\rho^{*}$ and $T^{*}$ are injective, this uniquely determines $A$. In this manner, we recover the superconnection $(\nabla, A)$ from the associated parallel transport.

Example. Let us conclude by considering the above construction in case that $M=$ $p t$, that is, $M$ is a point. The bundle $E$ together with the connection reduces in this case to a $\mathbb{Z} / 2$-graded vector space $V$, and the bundle endomorphism valued form 
$A$ reduces to an odd endomorphism $A \in \operatorname{End}^{1}(V)$. We have the pullback diagram

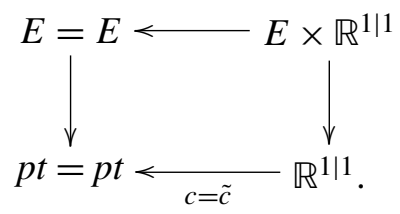

(It's enough to consider only this map, since the factor $S$ doesn't play a role here.) The pullback bundle is endowed with the trivial connection. The super parallel sections along $c$ are therefore given by the equation $D \psi=A \psi$.

Lemma 4.5. The solutions of the above equation are given by $(t, \theta) \mapsto e^{-t A^{2}+\theta A} v$ for some $v$ in $V$.

Proof. Indeed, we have

$$
\begin{aligned}
\left(\partial_{\theta}+\theta \partial_{t}\right) e^{-t A^{2}+\theta A} & =\left(\partial_{\theta}+\theta \partial_{t}\right)\left((1+\theta A) e^{-t A^{2}}\right) \\
& =A e^{-t A^{2}}+\theta e^{-t A^{2}}\left(-A^{2}\right) \\
& =A(1+\theta A) e^{-t A^{2}} \\
& =A e^{-t A^{2}+\theta A},
\end{aligned}
$$

where in the third equality we moved $A$ past $e^{-t A^{2}}$ without a sign change since $e^{-t A^{2}}$ is even, and past $\theta$ with a change of sign, since both $A$ and $\theta$ are odd. The lemma follows.

The parallel transport therefore defines a map

$$
\mathbb{R}^{1 \mid 1} \ni(t, \theta) \mapsto e^{-t A^{2}+\theta A} \in G L(V),
$$

which is in fact a supergroup homomorphism $\mathbb{R}^{1 \mid 1} \rightarrow G L(V)$ since composition on $\mathbb{R}^{1 \mid 1}$, which preserves the vector field $D$, corresponds to composition (multiplication) on $G L(V)$. For a direct proof of this see [Stolz and Teichner 2004].

\section{Acknowledgments}

I would like to thank especially Stephan Stolz for guidance and invaluable help, Nigel Higson for continuous encouragement, and Liviu Nicolaescu for stimulating discussions. This paper was prepared, in part, while the author was a Postdoctoral Scholar at Pennsylvania State University.

\section{References}

[Berline et al. 1992] N. Berline, E. Getzler, and M. Vergne, Heat kernels and Dirac operators, vol. 298, Grundlehren der Mathematischen Wissenschaften, Springer, Berlin, 1992. MR 94e:58130 Zbl 0744.58001 
[Bunke and Schick 2007] U. Bunke and T. Schick, "Smooth K-theory”, Preprint, 2007. arXiv 0707. 0046

[Deligne and Morgan 1999] P. Deligne and J. W. Morgan, "Notes on supersymmetry (following Joseph Bernstein)", pp. 41-97 in Quantum fields and strings: a course for mathematicians, I (Princeton, NJ, 1996/1997), edited by P. Deligne et al., Amer. Math. Soc., Providence, RI, 1999. MR 2001g:58007 Zbl 01735158

[Dumitrescu 2006] F. Dumitrescu, Superconnections and Parallel Transport, Thesis, University of Notre Dame, 2006, Available at http://etd.nd.edu/ETD-db/theses/available/etd-07212006-131339/.

[Freed 1999] D. S. Freed, Five lectures on supersymmetry, American Mathematical Society, Providence, RI, 1999. MR 2000h:58015 Zbl 0937.81001

[Hopkins and Singer 2005] M. J. Hopkins and I. M. Singer, "Quadratic functions in geometry, topology, and M-theory”, J. Differential Geom. 70:3 (2005), 329-452. MR 2192936 Zbl 05033792

[Kostant 1977] B. Kostant, "Graded manifolds, graded Lie theory, and prequantization", pp. 177306 in Differential geometrical methods in mathematical physics (Bonn, 1975), edited by K. Bleuler and A. Reetz, Lecture Notes in Mathematics 570, Springer, Berlin, 1977. MR 58 \#28326 Zbl 0358.53024

[Leĭtes 1980] D. A. Leĭtes, "Introduction to the theory of supermanifolds", Uspekhi Mat. Nauk 35:1(211) (1980), 3-57, 255. In Russian; translated in Russ. Math. Surv. 35:1 (1980), 1-64. MR 81j:58003 Zbl 0439.58007

[Manin 1988] Y. I. Manin, Gauge field theory and complex geometry, Grundlehren der Mathematischen Wissenschaften 289, Springer, Berlin, 1988. MR 89d:32001 Zbl 0641.53001

[Quillen 1985] D. Quillen, "Superconnections and the Chern character", Topology 24:1 (1985), 8995. MR 86m:58010 Zbl 0569.58030

[Šander 1980] V. N. Šander, "Vector fields and differential equations on supermanifolds", Funktsional. Anal. i Prilozhen. 14:2 (1980), 91-92. In Russian; translated in Functional Anal. Appl. 14:2 (1980) 160-162. MR 82c:58004

[Stolz and Teichner 2004] S. Stolz and P. Teichner, "What is an elliptic object?", pp. 247-343 in Topology, geometry and quantum field theory (Oxford, 2002), edited by U. Tillmann, London Math. Soc. Lecture Note Ser. 308, Cambridge Univ. Press, Cambridge, 2004. MR 2005m:58048 Zbl 05020127

[Varadarajan 2004] V. S. Varadarajan, Supersymmetry for mathematicians: an introduction, Courant Lecture Notes in Mathematics 11, New York University Courant Institute of Mathematical Sciences, New York, 2004. MR 2005g:58011 Zbl 02123108

[Witten 1999] E. Witten, "Homework, Problem FP2", pp. 609-717 in Quantum fields and strings: a course for mathematicians, I (Princeton, NJ, 1996/1997), Amer. Math. Soc., Providence, RI, 1999. MR 2000i:58037 Zbl 01735163

Received November 17, 2007.

FLORIN DUMITRESCU

DEPARTMENT OF MATHEMATICS

PENNSYlVANia STATE UNIVERSITY

UNIVERSITY PARK, PA 16802

UNITED STATES

dumitres@math.psu.edu 